3rd International Tribology Symposium of IFToMM, March in 2013, Luleå

\title{
Lubricating grease shear flow and boundary layers in a concentric
}

\section{cylinder configuration}

\author{
J. X. LI ${ }^{1}$, LARS G. WESTERBERG ${ }^{2}$, E. HÖGLUND ${ }^{1 *}$, T. S. LUNDSTRÖM ${ }^{2}$, P. M. LUGT ${ }^{3}$, \\ P. BAART ${ }^{1,3}$
}

1 Division of Machine Elements, Luleå University of Technology, SE-971 87 Luleå, Sweden 2 Division of Fluid and Experimental Mechanics, Luleå University of Technology, SE-971 87 Luleå, Sweden

3 SKF Engineering \& Research Centre, Nieuwegein, The Netherlands

\begin{abstract}
Grease is extensively used to lubricate various machine elements such as rolling bearings, seals, and gears. Understanding the flow dynamics of grease is relevant for the prediction of the grease distribution for optimum lubrication and the migration of wear- and contaminant particles. In this study grease flow is visualized using the method of micro Particle Image Velocimetry; the experimental setup comprises a concentric cylinder with rotating shaft to simulate the grease flow in a Double Restriction Seal (DRS) geometry with two different grease pocket heights. It is shown that grease may be partially yielded in the large grease pocket geometry and fully yielded in the small grease pocket geometry. For the small grease pocket geometry, it is shown that three distinct grease flow layers are present: a high shear rate region close to the stationary wall, a bulk flow layer, and a high shear rate boundary region near the rotating shaft. The grease shear thinning behaviour and its wall slip effects have been detected and discussed.
\end{abstract}

Key words: grease flow; micro Particle Image Velocimetry (0); boundary layer; velocity profile, lubrication 


\section{Introduction}

Lubricating grease is commonly applied to lubricate e.g. rolling bearings, seals and gears. It is a semi-solid material, which prevents it from easily flowing/leaking out from the bearing system and gives it sealing properties, protecting the system against ingress of contaminants. Compared to lubricating oil, grease shows much more complex rheology, which makes it more difficult to model and understand grease flow. Grease lubricated rolling bearings often run under starved elastohydrodynamic (EHD) lubrication conditions [1]. This means that the thickness of the lubricant film is mainly determined by the limited supply of lubricant to the contacts between rolling elements and bearing races. Grease near the rolling elements acts as a reservoir to lubricate the bearing. Lugt et al. [2] showed that the initial conditions determine the grease lubrication process and that a long grease life could be achieved by an optimum reservoir formation. This reservoir formation is determined by the flow of the grease during the so called 'churning phase'. It was also shown that the grease flow near the bearing rolling element-ring contacts plays an important role for the replenishment of these contacts.

The flow of grease is however not only determined by its rheological properties but also by the material and roughness of the elements carrying the grease, such as seals, cages, rolling elements and rings. In particular grease flow behavior close to surfaces of these elements has shown to be of interest and especially the existence and origins of wall slip. Bramhall and Hutton [3] and Vinogradov [4] have argued that wall slip occurs over a layer of oil due to the lower concentration of thickener particles at the wall. Bramhall and Hutton introduced a model assuming a low viscosity layer near to the wall and calculated a layer thickness of about $0.05 \mu \mathrm{m}$ for smooth lithium-based greases. Czarny [5] claims that there exists a concentration gradient of thickener close to the wall due to the interactions between the particles of the grease thickener and the wall which resulted in the formation of a thickener wall layer, with on top of this, an oil layer. He concludes that wall slip depends on the wall material and on the thickener type. Westerberg et al. [6] used $\mu$ PIV to investigate grease flow in a straight channel using side walls with different materials (steel and brass) and different roughness. They showed that wall slip primarily occurred for stiff greases and that the roughness of the surface had a negligible impact on the wall slip effect. However, their choice of roughness levels was given by values that can be found in rolling bearings and their surfaces were therefore much smoother than those generally applied in rheometers to prevent wall slip.

Two types of flow restrictions were applied into the straight channel in order to simulate the flow of grease near a seal pocket in Li et al. [7]. Later, in [8-9] a configuration comprising of a rotating shaft and two narrow gap sealing-like restrictions (a so called Double Restriction Seal, DRS) was designed to simulate the 
sealing contact of a labyrinth type of seal. $\mu$ PIV has proven to be an appropriate method to visualise the flow of grease in the previous work. The objective of this study is to investigate the grease flow in DRS geometries and discuss the wall slip effects at both the stationary wall and the rotating shaft.

\section{Methodology}

The experiments were carried out in a grease chamber, or grease pocket, between a rotating inner shaft and a stationary housing and two sealing restrictions (in the inlet and outlet of the chamber), see Figure B1. A detailed description of the setup can be found in Green et al. [8]. A steel ring with thickness $1.1 \mathrm{~mm}$ could be inserted into the grease pocket to reduce the pocket height between shaft and housing and therefore get different types of velocity profiles in the pocket. By using the ring, the height of the grease pocket changed from $1.5 \mathrm{~mm}$ to $0.4 \mathrm{~mm}$. The ring is made of steel, ground to a measured roughness value $(\mathrm{Ra})$ of $0.2 \cdot 10-6 \mathrm{~m}$ in the flow direction and $1.5 \cdot 10-6 \mathrm{~m}$ perpendicular to the flow direction.

The grease velocity profile was measured in the F2' plane using a $\mu$ PIV system consisting of a high-speed CCD camera, an optical microscope, a pulsed laser, and a computer to process the data; see Figure B2. The basic principle behind $\mu$ PIV is to measure the speed of tracer particles within a fluid (i.e. grease in this case) using a pulsed laser as the light source. By assuming that these particles move with the same speed as the flow speed, the fluid velocity is measured. The laser pulses are synchronised with the camera, resulting in a set of images taken with a certain frequency. The particle motion from one image frame to the next is tracked using a cross correlation technique, resulting in the direction and velocity of the particles and hence ultimately the motion of the grease. In this study fluorescent particles with a diameter of $3.23 \mu \mathrm{m}$ were used as tracer particles in the grease. The microscope lens used has a magnification of $10 \mathrm{X}$. For more details about the $\mu$ PIV method the reader is referred to previous work from the authors [8].

Three lithium greases with different consistency (NLGI grade) have been considered: NLGI 2, NLGI 1 and a NLGI 00 grease respectively. The NLGI 1 and NLGI 2 greases show shear-thinning properties with a non-zero yield stress value and a power law exponent smaller than 1, while the NLGI 00 grease shows an approximately Newtonian behavior. The details about the greases can be found in the authors' previous work [8]. 


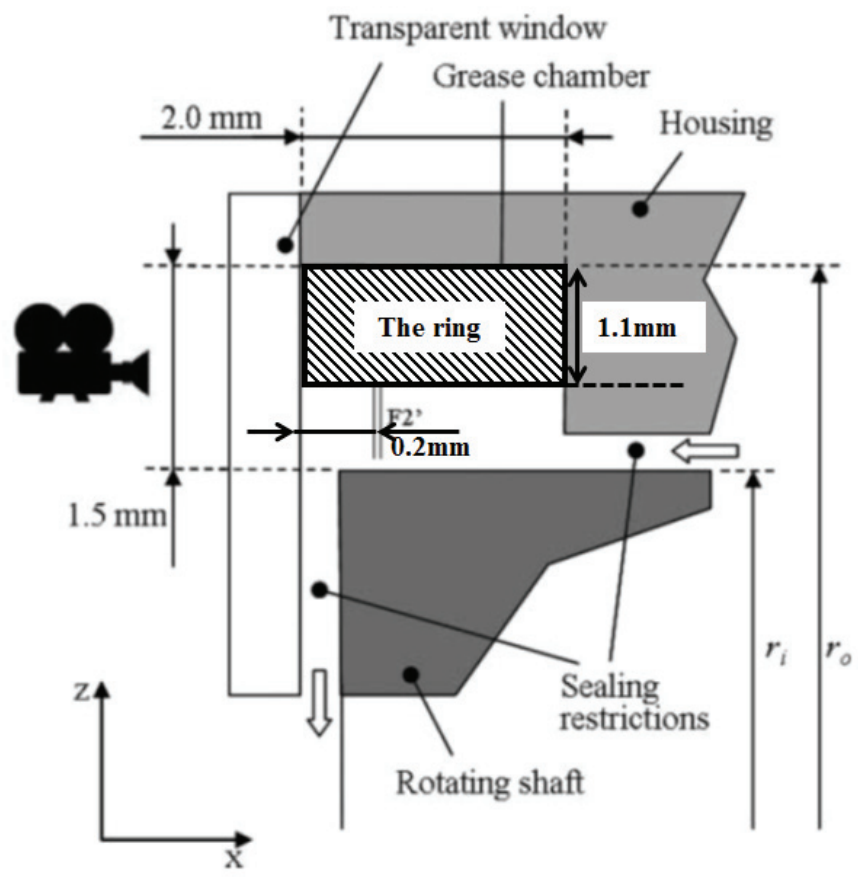

Figure B1. Grease chamber/pocket with ring in the DRS setup with the focus plane of the camera indicated by F2'.

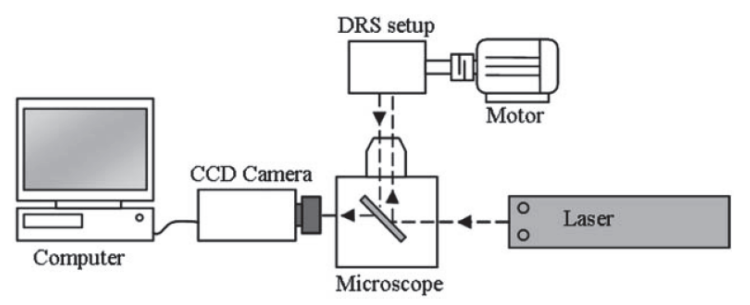

Figure B2. Setup of $\mu$ PIV system for DRS. From Green et al. [8].

\section{Results and discussion}

In this section the grease velocity profiles in the DRS grease chamber with two different pocket heights will be shown and investigated.

\subsection{Grease velocity profile and yield behavior in large grease pocket}

In Figure B3 (Baart et al. [9]) the velocity profiles for the case with a large grease pocket height are shown. Clearly, part of the grease, close to the stationary housing, is stationary and not flowing. This is called an un-yielded region where the shear stresses 
are lower than the yield stress. Closer to the rotating shaft the grease starts flowing implying a shear stress value above the characteristic yield stress value for the grease. Note that in the concentric cylinder rheometer, and the DRS setup, the shear stress is a function of the radial position and reduces at larger radii. In the yielded region the three greases show similar flow characteristics as for the case with ring, presented in the next section: the NLGI 2 and NLGI 1 greases have a curved velocity profile due to the shear thinning properties of the greases, while the NLGI 00 grease exhibits a more or less linear relationship between the shear rate and the shear stress, indicating a Newtonian rheology with a very low yield stress value. Furthermore, it is shown that for the same shaft speed of $0.02 \mathrm{~m} / \mathrm{s}$, the unyielded stationary part of the NLGI 2 grease extends further from the stationary housing than the corresponding region for the NLGI 1 grease. This result follows from the higher yield stress value compared to the NLGI 1 grease.

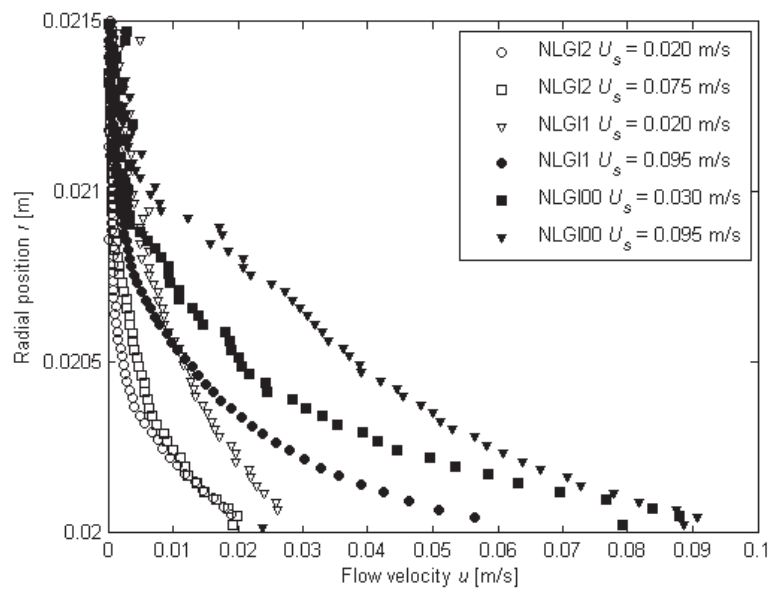

Figure B3. Velocity profile of the three greases in DRS with large gap[9]

\subsection{Grease velocity profile and shear banding in small height grease pocket}

Figures B4-B6 show that the NLGI 2, NLGI 1 and NLGI 00 greases are fully yielded in the case with the small grease pocket height for shaft speeds Us ranging from 0.020 $0.075 \mathrm{~m} / \mathrm{s}$ for NLGI 1 and NLGI 2 and $0.025-0.090 \mathrm{~m} / \mathrm{s}$ for NLGI 00. Compared to NLGI 00, the NLGI 2 and NLGI 1 greases have a more curved velocity profile or more pronounced shear thinning behaviour.

The wall slip layers are very thin and the velocity profiles in these layers have not been measured here. Instead, the layers are identified by extrapolation of the measured velocity profiles towards the walls and will be further discussed in section 3.4. In the absence of wall slip the extrapolation should lead to zero velocity at the housing 
surface and the shaft speed at the shaft surface. Figure B4 reveals that three different regions are present for the NLGI 2 grease velocity profile in addition to the wall slip layers: a relatively high shear rate region in the vicinity of the stationary housing wall (upper wall in Figure B1), a mid-region where the velocity increases continuously in the direction towards the moving shaft boundary, and thirdly a high shear rate region in the vicinity of the rotating shaft. These regions are also referred to as shear banding and also present for the NLGI 1 grease; for which its velocity profile is shown in Figure B5. Figure B6 shows that the NLGI 00 grease has an almost linear profile indicating a Newtonian rheology with the shear rate being proportional to shaft speed. The shear rate in the centre part of the velocity profiles from Figures 4-6 has been calculated and plotted in Figure B7 as a function of the apparent shear rate defined as the ratio of shaft speed and grease pocket height (the ideal-Newtonian shear rate). The apparent shear rate is proportional to the shaft speed, meaning that the shear forces increase with an increasing shaft speed, which in turn means that the viscosity of the grease decreases due to its shear thinning properties. In the case of Newtonian behaviour, the data points for the three greases in Figure B7 would show a straight line with slope 1 . This is clearly not the case where the deviation is more pronounced for the greases with higher NLGI numbers.

This shear banding effect was not observed earlier in the large pocket height. However, Figure B3 does show some indication of shear banding but not as obvious as with the smaller grease pocket height. Unlike the yield behaviour, which is a result of the varying shear stress over the pocket height (radius), the shear banding is not expected to depend on the geometry and is also expected to be present in other systems and rheometers with grease films of similar thickness. This result illustrates that an error is made in the calculation of the shear rate in rheometers which is always done through the apparent shear rate.

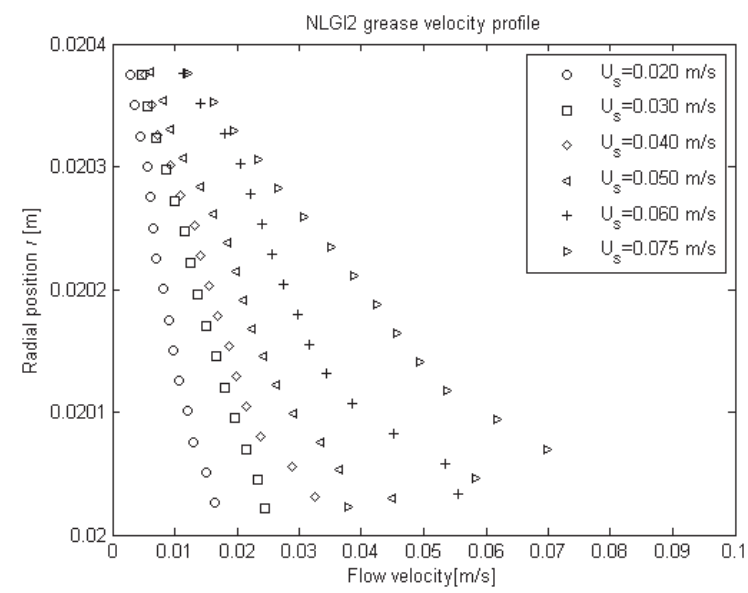

Figure B4. Velocity profile of the NLGI 2 grease in DRS with ring. $U_{\mathrm{s}}=$ shaft peripheral speed 


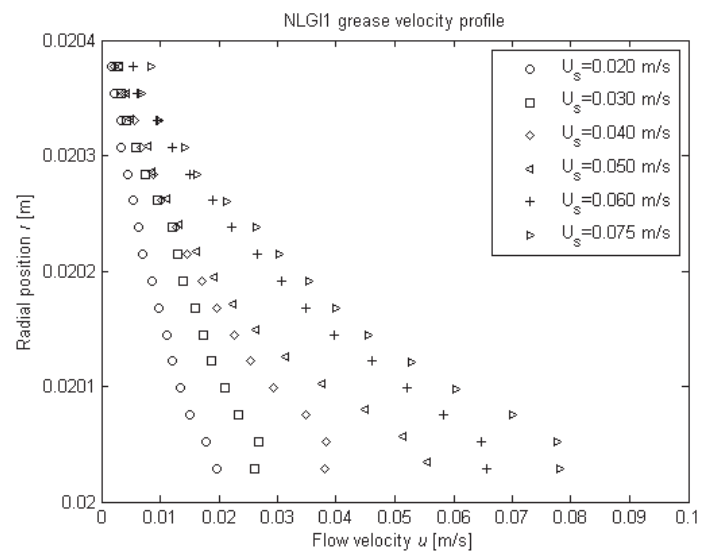

Figure B5. Velocity profile of the NLGI 1 grease in DRS with ring. $U_{s}=$ shaft peripheral speed

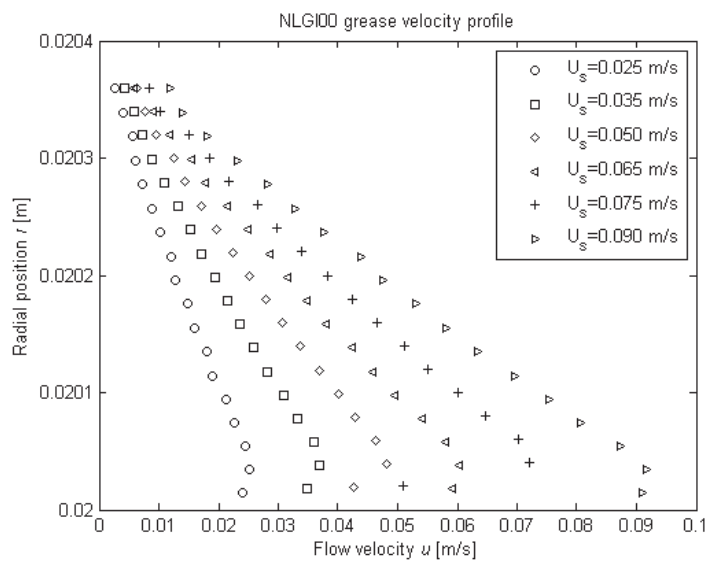

Figure B6. Velocity profile of the NLGI 00 grease in DRS with ring. $U_{s}=$ shaft peripheral speed

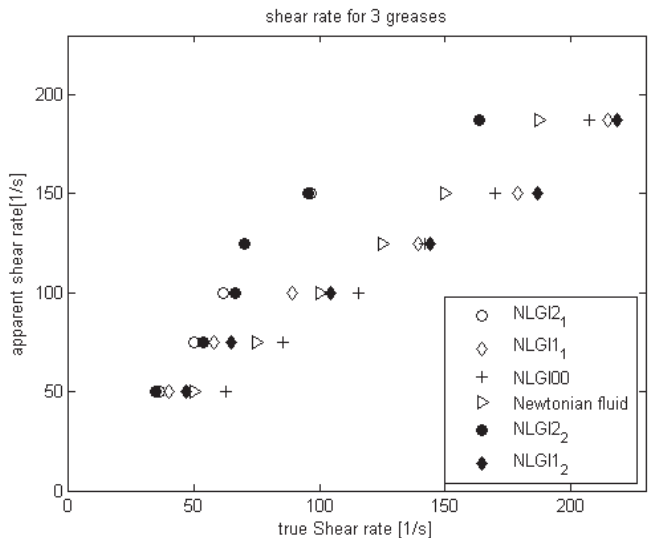

Figure B7. True shear rate calculated from the linear part in the velocity profiles for the three greases versus the apparent shear rate defined as the ratio of shaft speed and gap height. Black filled symbols for repeated test of NLGI 1 and NLGI 2 greases. 


\subsection{Comparison of NLGI 2 grease velocity profiles in the DRS with small and large pocket height}

The NLGI 2 grease velocity profiles as a function of the dimensional radial position are shown in Figures B8 and B9 where in Figure B9 the radial position has been made dimensionless using the ratio of radial position and pocket height. In Figure B8 the red dotted line indicates the position of the ring. Here the grease velocity profile for the case with ring is almost following the corresponding velocity profile for the case without ring at the lower shaft speed. The profile has a more curved form for the lower shaft speed, while it is more linear at a higher shaft speed comparing the cases with small and large pocket height. In Figure B9, it is apparent that the grease is partially yielded in the grease pocket with large pocket height, and that the yield point moves away from the rotating shaft (towards to the upper housing wall) as the shaft speed increases from 0.02 to $0.075 \mathrm{~m} / \mathrm{s}$, while the grease is fully yielded for the case with ring. Here the reduced pocket height due to the insertion of the ring in the DRS geometry implies that the shear stress value throughout the region exceeds the yield stress. When the rotational speed is high enough the induced shear forces in the grease will exceed the yield stress and the grease will be fully yielded with moving grease in the whole pocket as a result. A similar effect was shown earlier in [7] where the flow depth increased due to higher velocities (and therefore shear) caused by an increased pressure drop for the straight channel with double restrictions. Another example is the work from Westerberg et al. [6] where the plug flow changed into a more parabolic velocity flow as the velocities and therefore shear forces increased.

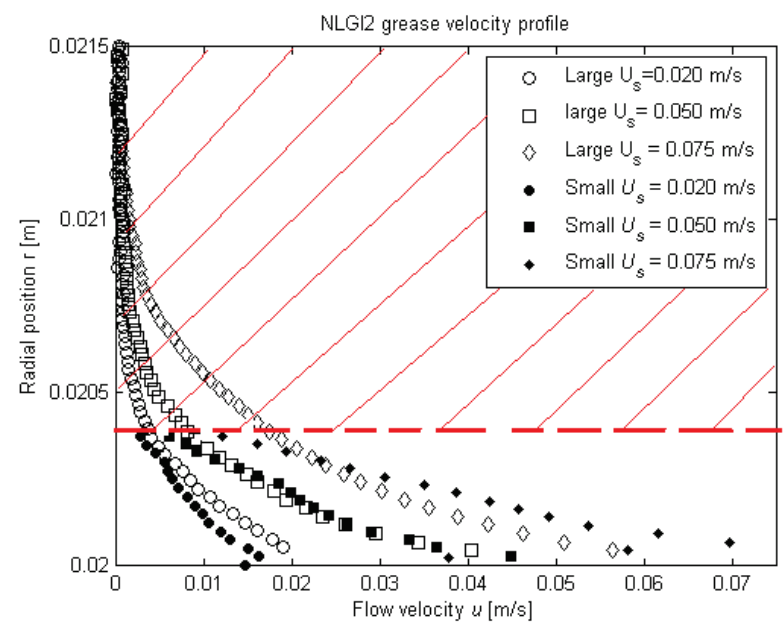

Figure B8. Velocity profile of the NLGI 2 grease in DRS with large and small gap. 


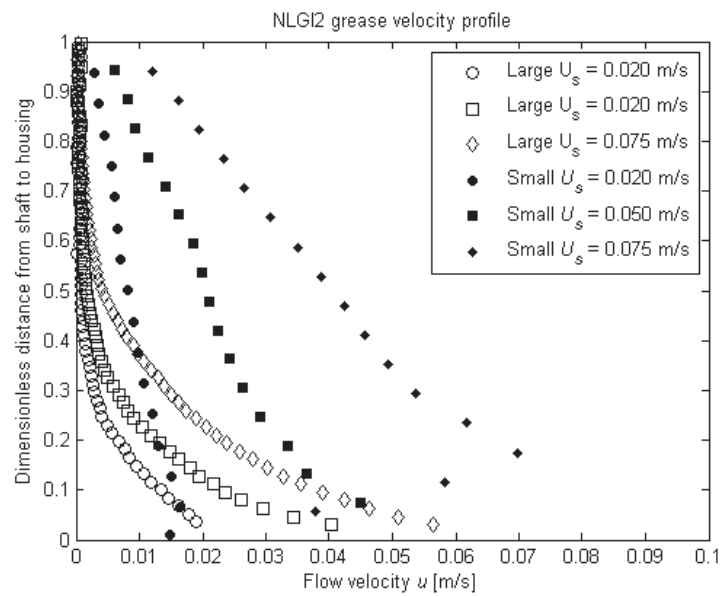

Figure B9. Velocity profile of the NLGI 2 grease in DRS with large and small gap, and dimensionless radial distance from the rotating shaft.

\subsection{Wall (slip) effects in DRS with small gap}

Wall slip effects for grease flow have been addressed by several authors throughout the years $[3,4,5,6]$, where also Westerberg et al. [6], also used the method of $\mu$ PIV to visualize the grease velocity profile. In the present study, the velocity profile close to the stationary housing boundary (upper boundary in Figure B1) is shown in Figure B10 where the dimensional velocity is plotted as a function of the radial co-ordinate and in Figure B11, where the corresponding dimensionless velocity is plotted. In these figures the area close to the wall is zoomed-in to highlight wall effects. Figures B10B11 show that a slip layer is present in the region stretching from the stationary housing boundary wall $(\mathrm{y}=0)$ to the location of the first data point (y about $20 \mu \mathrm{m})$. As mentioned above, the existence of a slip layer is indicated by a non-zero extrapolated velocity on the stationary housing. The figure shows that the wall-slip velocity for the NLGI2 grease is higher than for the NLGI 1 grease, which is ascribed to the lower base oil viscosity of the NLGI2 grease. The result coincides with the results from Westerberg et al. [6]. In the present study it is surprising that the slip velocity for the NLGI 00 grease is higher than for the NLGI 1 grease. The same behaviour has been detected in [6] when using a rougher brass wall with a Ra value of $5.41 \mu \mathrm{m}$. 


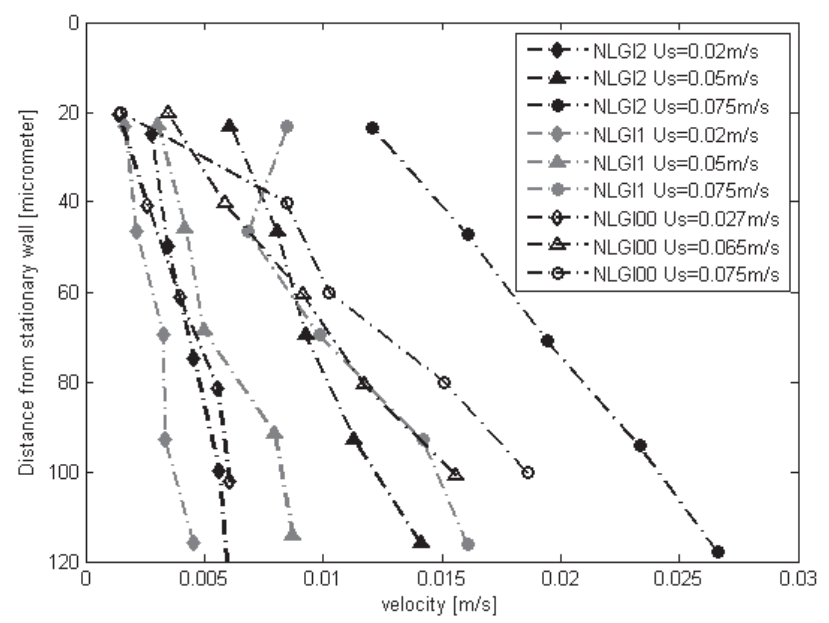

Figure B10. Velocity profiles close to the stationary housing wall for the three greases.

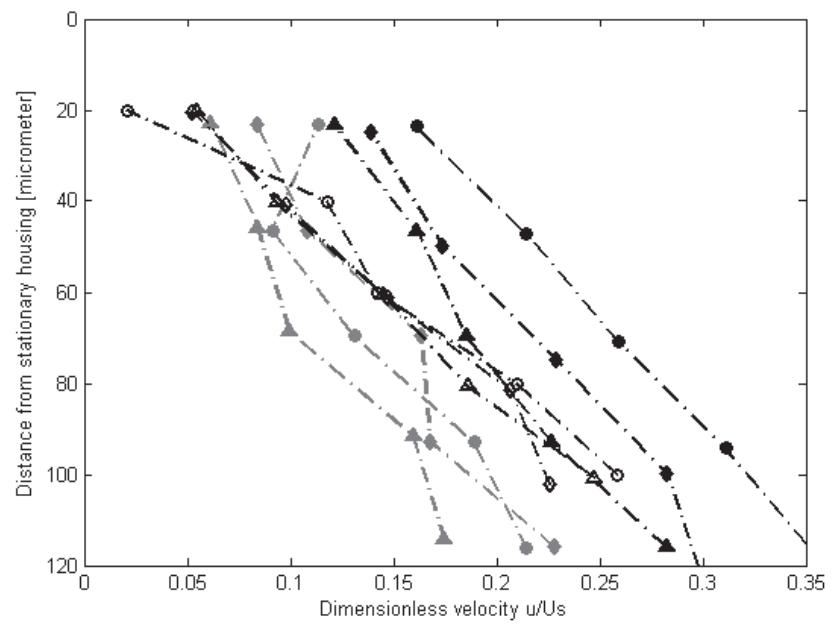

Figure B11. Dimensionless velocity profiles close to the stationary wall for the three greases $\left(u / U_{s}\right.$, $u$ is velocity of grease and $U_{s}$ is shaft speed); $\diamond$ low shaft speed $0.02 \mathrm{~m} / \mathrm{s}(0.027 \mathrm{~m} / \mathrm{s}$ for NLGI 00 grease), $\Delta$ medium shaft speed $0.05 \mathrm{~m} / \mathrm{s}(0.065 \mathrm{~m} / \mathrm{s}$ for NLGI 00 grease), $\circ$ high shaft speed $0.075 \mathrm{~m} / \mathrm{s}$; no fill for NLGI 00, grey for NLGI 1 and black for NLGI 2 grease.

\section{Summary and Conclusions}

Grease velocity profiles have been measured in a grease pocket which resembles a concentric cylinder configuration. For the large pocket height, an un-yielded region appears near the stationary wall where the grease behaves as a solid body, indicating the presence of a yield stress. It is also shown that the position where the yield stress is exceeded approaches the stationary wall with increasing shaft speed. 
Wall slip phenomena have been observed near the stationary wall in the case of a small pocket height. The wall-slip velocity at the stationary wall is highest for NLGI 2 grease which has the lowest base oil viscosity.

For the small pocket height geometry, it is shown that there is grease flow across the whole height, i.e., the yield stress of the grease is exceeded throughout the pocket. Furthermore, three distinct grease flow layers are present in addition to the wall slip effects, a high shear rate region close to the stationary wall, a bulk flow layer, and a high shear rate boundary region near the rotating shaft. As a result, a clear deviation between the ideal-Newtonian shear rate and the real shear rates in the grease was measured for the NLGI 1 and NLGI 2 greases. Attention should be paid to this effect when evaluating grease rheology in rheometer applications.

\section{Acknowledgements}

The authors would like to thank the Swedish Science Council (VR) for their financial support for the work presented in this paper and thank Alexander de Vries, Director SKF Group Product Development, for his kind permission to publish this paper.

\section{References}

[1] Cann, P.M.E., Thin-film grease lubrication, Proceedings of the Institution of Mechanical Engineers Part J-Journal of Engineering Tribology (1999); 213: 405-416.

[2] Lugt, P. M., Velickov, S., \& Tripp, J. H. (2009). On the chaotic behavior of grease lubrication in rolling bearings. Tribology Transactions, 52(5), 581-590

[3] Bramhall, A. D., \& Hutton, J. F. (1960). Wall effect in the flow of lubricating greases in plunger viscometers. British Journal of Applied Physics, 11(8), 363-371.

[4] Vinogradov, G. V., Froishteter, G. B., \& Trilisky, K. K. (1978). The generalized theory of flow of plastic disperse systems with account of the wall effect. Rheologica Acta, 17(2), 156-165.

[5] Czarny, R. (2002). The influence of surface material and topography on the wall effect of grease. Lubrication Science, 14(2), 255-274

[6] Westerberg, L. G., Lundström, T. S., Höglund, E., \& Lugt, P. M. (2010). Investigation of grease flow in a rectangular channel including wall slip effects using microparticle image velocimetry. Tribology Transactions, 53(4), 600-609.

[7] Li, J. X., Höglund, E., Westerberg, L. G., Green, T. M., Lundström, T. S., Lugt, P. M., \& Baart, P. (2012). $\mu$ pIV measurement of grease velocity profiles in channels with two different types of flow restrictions. Tribology International, 54, 94-99.

[8] Green, T. M., Baart, P.,Westerberg, L. G., Lundström, T. S., Höglund, E., Lugt, P. M., and Li, J. X. (2011), “A New Method to Visualize Grease Flow in a Double 
Restriction Seal Using Micro Particle Image Velocimetry," Tribol. Trans., 54(5), pp 784-792.

[9] Baart, P., Green, T. M., Li, J. X., Lundström, T. S., Westerberg, L. G., Höglund, E., \& Lugt, P. M. (2011). The influence of speed, grease type, and temperature on radial contaminant particle migration in a double restriction seal. Tribology Transactions, 54(6), 867-877.

[10] F.A.Morrison, ch.1 in "Understanding Rheology", Oxford University Press, 2001 [11] T.M.Green, PIV in practice, Doctoral thesis, Sweden: Luleå University of Technology; 2011, ISBN: 978-91-7439-313-2. 